\title{
The Implementation of the Feedback Principle in Science and Technics
}

\author{
Anna L. Buran ${ }^{1, *}$, Vladislav E. Kozlov ${ }^{1}$, and Andrey V. Filyukov ${ }^{1}$ \\ ${ }^{1}$ National Research Tomsk Polytechnic University, 634050 Tomsk, Russia
}

\begin{abstract}
In this study positive and negative influence of the feedback have been examined. A feedback principle in the alternating current amplifier and in the speed regulator of the turbine rotation was described. The main conclusion of the positive and negative effect was obtained. Negative feedback worsens the properties of an object, reducing a strengthening factor, delaying regulatory action. Positive feedback considerably raises device work stability. This research will enable to identify the importance and effectiveness of the feedback principle in science and technics.
\end{abstract}

\section{Introduction}

Scientists and researchers refer a feedback principle to one of the fundamental and universal principles. Some scientists present robust overlapping load frequency output feedback control of multi-area interconnected power systems [1], others develop an integrated guidance and feedback control scheme for steering an underactuated vehicle through desired waypoints in three-dimensional space [2] and investigate active disturbance attenuation control for permanent magnet synchronous motor via feedback domination and disturbance observer [3]. The aim of this work is to study the effect of feedback principle in science and technics, using the examples of various technical devices, such as an operational amplifier and a regulator of turbine speed. It is assumed that no system exists without feedback. The system, which doesn't have feedback degrades and collapses. Feedback can be positive and negative, in both cases a feedback problem is to return the system to an optimum condition. To solve a problem with control means to support an operating object into a desirable condition. The analysis of control processes in various areas, allows to establish that control, irrespective of kinds of objects, is always carried out according to a feedback principle. There can be control processes in technics, in society and in nature and in education. Some researchers refer to potential of case technology in technics [4], others prove that control of different objects is carried out according to one principle, that's why all control systems must have common features.

\section{A feedback principle in the alternating current amplifier}

\footnotetext{
*Corresponding author: aburan@tpu.ru
} 
Regarding the above confirmed theory, the characteristics of an operational amplifier should be studied carefully. At an amplifier output the divider of voltage R1-R2 is put, and the part of target voltage moves back to an amplifier input. There is an input voltage $u$ input at an amplifier input, which is necessary to enhance, and feedback voltage $U$ f.b. If these types of voltage are added up, feedback is called positive if they are subtracted, it's negative.

For the purpose of this research, we should know about negative feedback. At an amplifier input the difference of voltage $u$ input - uo.c is put. This difference amplifies in Ko times. The achieved result is an amplifier target voltage.

The results indicated that negative feedback lowers proportional-action coefficient. It will be provided after considering a numerical example. A proportional-action coefficient without feedback $K_{\mathrm{o}}$ is 100 and the feedback factor $\beta=50$. Then to the amplifier with feedback we will get: $K=100 / 1+0.5 \cdot 100=100 / 51 \approx 1.96$

The strengthening factor has decreased exactly in 51 times. There is a reasonable question: what such feedback, which worsens amplifier characteristics is necessary for. But, losing in strengthening, we can get another advantage. It's negative feedback, which raises the stability of amplifier work. Regarding this issue we assume that for any reasons the factor of strengthening $K_{\mathrm{o}}$ has increased by $20 \%$ and $K_{\mathrm{o}}=120$, instead of 100 , as we thought. The results indicated that the amplifier without feedback output voltage will also increase by $20 \%$. Now we will see what will happen, if the amplifier is captured by negative feedback: $K=120 / 1+0.5 \cdot 120=120 / 61=1.97$. We achieved the result that gain constant has changed very little.

So, negative feedback reduces amplifier coefficient, but it increases stability of amplifier work. We will come back to strengthening factor with negative feedback: $K=K_{\mathrm{o}} / 1+\beta \cdot K_{\mathrm{o}}$. At that time we assumed that product $\beta \cdot K_{\mathrm{o}}$ is much more than one. Then it is possible to consider approximately: $K=K_{\mathrm{o}} / \beta \cdot K_{\mathrm{o}}=1 / \beta$.

We see that proportional-action coefficient $K_{\mathrm{o}}$ is absent in our formula. It follows that if values $\beta$ and $K_{\mathrm{o}}$ are chosen correctly, changes of transistor parameters or other elements of the amplifier don't influence on the strengthening value. Work stability of the scheme depends only on the feedback circuit stability. In feedback circuits there are no transistors or any other elements, which change their parameters considerably. That's why the factor $\beta$ can be supported with great accuracy and consequently the whole amplifier will work precisely.

There are also other important advantages which have the amplifiers with negative feedback. One of them influences on the size of input resistance of the amplifier. Good amplifier should have high resistance. Such amplifier allows to intensify lower power signals very much and doesn't deform operating mode of electronic devices.

So negative feedback increases the amplifier input resistance at $\left(1+\beta \cdot K_{0}\right)$ times. These are not the only advantages of negative feedback. It's found that in the amplifiers captured by the feedback input resistance reduces with $\left(1+\beta \cdot K_{\mathrm{o}}\right)$ time. The amplifier with small input resistance can work at any loading. Thus, the size of load resistance doesn't render the influence on amplifier work.

\section{A feedback principle in speed regulator of the turbine rotation}

The energy resources are frequently wasted in Russia [5]. Regarding this issue, we will find out the technology of getting electrical energy at the Russian thermal power station using the coal. Large pieces of coal are transformed into coal dust. This dust is blown by a stream of hot air into a furnace through special burners. The flame heats up water in thin tubes which cover furnaces' walls and the heated top internal gases volatilize through the chimney. At first they meet water tubes, transforming warm water into steam. Then gases 
are supplied to a special device - economizer, warming cold water in it and refilling water in the furnace, and, at last, - in air heater. The fuel burns, having transferred heat to water. The water in a furnace has been heated up and has vaporized, but this steam is not hot enough, cooling down, it will turn to water drops quickly. That's why, first of all, steam gets into superheater coil pipes where steam is heated up to very high temperature. This steam goes into turbines through steam pipes. Fresh "sharp" steam from a furnace is under the pressure of 150-200 atm. It passes through stationary blades. Shovels of the first row of a rotor start moving. Then steam gets to channels of the first diaphragm. And again the part of its pressure transforms into speed, and this speed works well together on the second blade tip. This repeats 10-12 times till steam pressure becomes moderate. So, initial pressure is used up smoothly and sparingly.

After that the steam goes to the condenser where cold water circulates continuously through thin brass tubes. It cools steam and transforms it into water, which is called condensate. A lot of water is provided by the circulating pump. So, steam is formed, having turned to condensate - very pure water which does not contain harmful chemical or mechanical impurities. Such clear water comes back from the condenser into a copper with the help of special pumps. The results indicated that the powerful steam turbine has a speed of $3000 \mathrm{rpm}$ and it rotates a rotor of the electric generator and due to it in a winding stator the generator (a motionless part) according to the law of electromagnetic induction forms the alternate current with a frequency of $50 \mathrm{~Hz}(3000 \mathrm{rpm}$ equals $50 \mathrm{about} / \mathrm{c})$ and pressure from 6 to $20 \mathrm{sq}$. Regarding this issue, it can be concluded from research that electric energy is the main "product" of a power station.

Regarding the process of frequency regulation, it's assumed that speed regulators consist of the following basic parts: instrument movement centrifugal pendulum, having a role of instrument movement; a sliding piston; the oil engine and the wave of rotary axis speed.

The speed regulator works in the following way: pendulum spheres move to each other after frequency falling, that's why the instrument movement descents and occupies position $\mathrm{A}^{*}$. As B and $\mathrm{G}$ are fixed, so the lever arm A-B rotates about point B and lever arm $\mathrm{G}-\mathrm{E}$ rotates about point $\mathrm{G}$. Consequently, buckets of sliding piston go down and open the windows, following which pressure oil comes to lower bucket separation of oil engine and it goes up. This bucket is connected to valves. When the engine bucket moves up, admission of propellant factor and rotor speed is rising, so point $\mathrm{A}$ of the instrument movement goes up too.

Simultaneously with moving of the oil-engine piston lever arm A-B turns round the point $\mathrm{A}$ and lifts up hinged points $\mathrm{B}, \mathrm{D}, \mathrm{E}$ and sliding piston together. This action is called as disengagement. Due to this, slide windows close earlier than rising of propellant factor inlet attacks. If there were no disengagement, sliding piston would rotate around the neutral location and value of rotor speed would change periodically. Since control process ends, when points B, D, E return to starting point, so the new location of the oil engine bucket fills with a new point A position. The more inlet of the moving factor we have the lower is the new position of the point $\mathrm{A}$, the less is turbine speed rotation.

The results indicated that, the peculiarity of regulators of turbine frequency rotation is the necessity of maintenance of rather considerable efforts for changing the position of steam turbines regulating valves or the hydraulic turbines directing device. And moving on these regulating elements should be carried out with great accuracy. Now the hydraulic engines often named in the systems of regulation as servo motors are used everywhere to solve the problem mentioned above. For the elements which help form the certain control mechanical, hydraulic and electric elements can be used. For example, we take the hydraulic servomotor operated by valve. Oil is brought in a valve's axle box under the pressure of $\mathrm{PH}$. In a neutral starting position valve's pistons block the channels connecting 
valve's axle box with the top and bottom cavities of the hydraulic engine. These cavities are filled with oil. At displacement of valve's pistons, for example downwards, the bottom cavity of the engine cylinder connects with a pressure line (pressure $\mathrm{PH}$ ), and the top one with a line of drain (pressure PC). The engine piston will move upwards until valve's pistons don't have neutral position or till the engine piston doesn't get its ultimate position. For replacement of the hydraulic engine by a link in the block diagram of the regulatory system and giving a definition of its transfer function, we will take the management connecting the expense of oil and moving of the engine piston during a short period of time $\Delta \mathrm{t}: S \Delta \mu=n K_{v}\left(P_{I}-P_{N}\right) \Delta t$ where $\Delta \mu$ - moving of the piston of the engine, $n-$ open section of valve's windows, $K v$ between the pressure and speed of oil flowing, $S$ - the area of the engine piston, accepted for simplification of the identical one from the top and bottom cavities. For a differential short period of time: $S \cdot d \mu / d t=n \cdot K_{v}\left(P_{\mathrm{n}}-P_{\mathrm{c}}\right) \mathrm{We}$ will come to relative units in (1), having accepted as basic values: $\mu$ max - the maximum working piston stroke of the engine, nmax - the area of cross-section section of completely open valve's windows. Then $T_{\mathrm{d}} \cdot(d \mu * / d t)=n^{*}(2)$, where $T_{\mathrm{d}}=S \cdot \mu_{\max } / n_{\max } \cdot K_{v} \cdot\left(P_{\mathrm{n}}-P_{\mathrm{c}}\right)-$ the time constant of a hydraulic engine. During $T_{\mathrm{d}}$ the engine piston moves from one ultimate position to another with fully opened valve's windows. According to the transferring function of the hydraulic engine is $W_{\mathrm{d}}(\mathrm{p})=1 / T_{\mathrm{d}}{ }^{*} p$ - that means the hydraulic engine can be presented as an integrating link. The time constant of $T_{\mathrm{d}}$ defines the speed of the regulatory system. The decrease at $\left(T_{\mathrm{a}}\right)$ action of a regulator towards power decrease is especially important. For example, it is required for steam turbines, tend towards closing to be about 0.2 seconds. For getting such short amount of time it is required the pump to be very productive to deliver liquid into a servo motor. As the great speed of the piston movement is reached mainly by closing of valves it is practically provided, not with the pump productivity increases, but using a unilateral servo motor which has oil pressure only while opening, and by force of a spring when closes.

Deng, W. \&Yao, J. analyze in their research adaptive integral robust control and application to electromechanical servo systems, giving experimental results, proved the of the effectiveness of the proposed controllers [6].

Yang, WH. considers that computer simulation technology can play an important role in the teaching reform of power system protective relaying course [7].

Depending on particular conditions and requirements of rotation frequency regulator a servo motor can be supplemented with rigid, flexible or combined feedback. Feedback can be executed with the help of different devices. As the ways of technical realization of feedback aren't principal while taking into consideration the processes of frequency and capacity regulation, we will take only the influence of kinds and parameters of feedback elements on hydraulic servo motor transferring function [8].

Regarding the servo motor with rigid negative feedback it can be concluded that at any transferring function of feedback link is $\left[W_{\mathrm{d}}(\mathrm{p})=1 / T_{\mathrm{d}}{ }^{*} \mathrm{p}+W_{\mathrm{oc}}(\mathrm{p})\right]$. Transferring function of a feedback link is $\left[W_{\mathrm{oc}}(\mathrm{p})=K_{\mathrm{oc}}\right]$, and transferring function of a servo motor with such feedback is $\left[W_{\mathrm{d}}(\mathrm{p})=1 / K_{\mathrm{oc}} *\left(T_{\mathrm{d}} / K_{\mathrm{oc}} * \mathrm{p}+1\right)\right]$. With rigid negative feedback the servo motor is an inertial link of the first order with time constant: $\left[T_{\mathrm{d}} / K_{\mathrm{oc}}\right]$ and strengthening factor: $\left[K=1 / K_{\mathrm{oc}}\right]$.

\section{Conclusion}

The paper has described the importance of the feedback principle in science and technics. Giving examples of two different technical devices the basic regularities for feedback have been revealed. Negative feedback worsens the properties of an object, reducing a strengthening factor, delaying regulatory action a bit. Positive feedback considerably raises 
device work stability. This research will enable to identify the importance and effectiveness of the feedback principle.

\section{References}

1. A. Ahmadi, M. Aldeen, Int. J. Electrical Power Energy Systems, 89, 156 (2017)

2. S.P. Viswanathan, A.K. Sanyal, E. Samiei, J. Intell. Rob. Systems: Theory Appl. (2017)

3. X. Niu, C. Zhang, H. Li, IET Control Theory and Applications, 11 (6), 807 (2017)

4. Z. Fedorinova, V. Vorobeva, M. Malyanova, XV International Conference Linguistic And Cultural Studies: Traditions And Innovations. Procedia - Social and Behavioral Sciences, 206, 247 (2015)

5. A. Buran, G. Nizkodubov, I. Pryahin, MATEC Web Conf. 91, 01054 (2016)

6. W. Deng, J.Yao, ISA Transactions 67, 256 (2017)

7. WH.Yang, 2016 International Symposium On Computer, Consumer And Control (Is3c), (2016)

8. S.A. Stavitsky, N.E. Palukhin, Y.V. Kobenko, E.S. Ryabova, EPJ Web Conf. 110, 01059 (2016) 